\title{
Tumor cell adhesion and migration supported by interaction of a receptor-protease complex with its inhibitor
}

\author{
Edgar G. Fischer, ${ }^{1}$ Matthias Riewald, ${ }^{2}$ Hui-Yu Huang, ${ }^{2}$ Yohei Miyagi, ${ }^{3}$ Yoshinobu Kubota, ${ }^{3}$ \\ Barbara M. Mueller, ${ }^{2}$ and Wolfram Ruf ${ }^{2}$ \\ ${ }^{1}$ Department of Pathology, University of Erlangen-Nürnberg, 91054 Erlangen, Germany \\ ${ }^{2}$ Departments of Immunology and Vascular Biology, The Scripps Research Institute, La Jolla, California 92037 USA \\ ${ }^{3}$ Departments of Pathology and Urology, Yokohama City University, Yokohama 236-0004, Japan \\ Address correspondence to: Wolfram Ruf, Departments of Immunology and Vascular Biology, IMM-17, \\ The Scripps Research Institute, 10550 North Torrey Pines Road, La Jolla, California 92037, USA. \\ Phone: (858) 784-2748; Fax: (858) 784-8480; E-mail: ruf@scripps.edu. \\ Edgar G. Fischer's present address is: Department of Pathology, University of New Mexico Health Science Center, \\ Albuquerque, New Mexico 87131, USA. \\ Received for publication July 2, 1999, and accepted in revised form September 23, 1999.
}

Tissue factor (TF), the cell-surface receptor for coagulation factor VIIa, supports metastasis. Equally important for this process are (a) interactions of the TF cytoplasmic domain, which binds the mobility-enhancing actin-binding protein 280 , and (b) the formation of a proteolytically active TFVIIa complex on the tumor cell surface. In primary bladder carcinoma cells, we find that this complex localizes to the invasive edge, in proximity to tumor-infiltrating vessels that stain intensely for TF pathway inhibitor (TFPI-1), the major inhibitor of the protease activity of the complex. In culture, binding of VIIa to TF-expressing tumor cells is sufficient to allow cell adhesion, migration, and intracellular signaling on immobilized TFPI-1. Immobilized heparin, a mimic for extracellular matrix-associated proteoglycans, binds physiological concentrations of TFPI- 1 in a conformation that supports TF-VIIa-dependent cell adhesion. Consistent with a functional role of TFPI-1 in complex extracellular matrices, we show that TF cooperates with integrin-mediated adhesion and migration on composite matrices that contain ligands for both integrins and the TF-VIIa complex. This study thus provides evidence for a novel mechanism of protease-supported migration that is independent of proteolytic matrix degradation but rather involves protease-dependent bridging of TF's extracellular domain to an ECM-associated inhibitor.

J. Clin. Invest. 104:1213-1221 (1999).

\section{Introduction}

Regulated pericellular proteolytic systems, consisting of proteases, specific cell-surface receptors, and inhibitors, promote tumor invasion and metastasis by degrading matrix barriers and by modulating cellular functions (1-3). Certain components of these systems are produced by the tumor cells themselves, whereas others either are contributed by tumor-associated stromal and inflammatory cells or extravasate from the blood plasma. Tissue factor (TF) is the cellular receptor and catalytic cofactor for the serine protease coagulation factor VIIa (VIIa). The cell-associated TF-VIIa complex is the major initiator of the coagulation pathways in vivo (4). TF is upregulated in a variety of malignancies (5). Its expression in epithelial tumors strongly correlates with fibrin deposition in the tumor stroma (6), reflecting activation of coagulation in the perivascular space around hyperpermeable tumor vessels (7).

The proteolytic function of TF-VIIa is regulated by the endothelium-derived TF pathway inhibitor (TFPI-1) that consists of 3 Kunitz-type inhibitory domains and a $\mathrm{COOH}$-terminus that is rich in basic amino acid residues (8). The first Kunitz domain binds to the catalytic site of VIIa, and the second binds to the active site of factor Xa. TFPI-1 typically locks TF-VIIa in a stable quaternary complex with factor Xa by simultaneously interacting with the active site of both proteases (8). A homologous Kunitz-type inhibitor, TFPI-2 (9, 10), inhibits TF-VIIa, but TFPI-2's second Kunitz-type domain does not bind factor $\mathrm{Xa}$ (11). The interaction of TFPI-2 with TF-VIIa is enhanced by heparin (11), but a physiological role of TFPI-2 in regulating function of the TF-VIIa complex has not been shown.

Experimental models of hematogenous metastasis demonstrate that TF has prometastatic function that depends on both signaling of the TF cytoplasmic domain $(12,13)$ and extracellular proteolytic activity of the TF-VIIa complex (13). The TF cytoplasmic domain interacts with actin-binding protein 280 (ABP-280; nonmuscle filamin) (14) that influences cell motility (15). Surrogate ligands, such as immobilized mAb's to TF, support tumor cell adhesion and migration, and ABP-280 is recruited to these TF-mediated matrix con- 
tact sites (14). By influencing tumor cell migration along with the extracellular activation of the coagulation cascade, TF shares the features of other cellular receptors that are implicated in the proteolytic modification of the tumor environment. Among those, the receptor for the serine protease urokinase serves as an adhesive receptor for vitronectin (16), and the integrin $\alpha_{v} \beta_{3}$ not only supports migration on various RGD motif-containing matrix proteins, but also binds matrix metalloproteinase-2 (MMP-2) to facilitate matrix degradation at the invasive edge (17).

Although ligation of the TF extracellular domain supports cell migration in in vitro assays, it is unclear whether relevant extracellular interactions of TF can support similar processes in vivo. This report demonstrates that at the invasive edge of human bladder cancer, the TF-VIIa complex forms in close proximity to its inhibitor TFPI-1 that is expressed on tumor-associated vessels. By in vitro studies, immobilized TFPI-1 is shown to support tumor cell adhesion and migration, and to elicit intracellular signaling that requires binding of VIIa to TF. Physiological concentrations of TFPI1 cooperate with integrin function in mediating tumor cell adhesion and migration. This study thus identifies a novel mechanism in which protease-mediated bridging of the cell-surface protease-binding receptor TF to an immobilized inhibitor can induce migratory responses of potential relevance for tumor invasion in the context of vascular hyperpermeability.

\section{Methods}

Cell adhesion assay. Nontissue culture-treated dishes were coated with the indicated concentrations of poly-L-lysine, fibronectin, anti-TF mAb TF9-6B4, unfractionated heparin (Elkins-Sinn, Inc., Cherry Hill, New Jersey, USA), or recombinant TFPI-1 (18) (kindly provided by A. Creasy, Chiron Corp., Emeryville, California, USA) overnight at $4{ }^{\circ} \mathrm{C}(14)$. To coat a mixture of TFPI- 1 and fibronectin, both were simultaneously added to the plates. Cells were serum starved overnight and harvested with trypsin/EDTA. Trypsin was quenched with soybean trypsin inhibitor $(1 \mathrm{mg} / \mathrm{mL})$, and cells were washed with serum-free medium (1\% BSA). After a 30-minute rest at room temperature, cells were seeded onto the coated, BSA-blocked plates. Heparin $(5 \mathrm{U} / \mathrm{mL})$, wild-type or mutant recombinant VIIa $(10 \mathrm{nM})$, or factor Xa $(10 \mathrm{nM})$ was added to the cell suspension before plating, as indicated. The inhibitory anti- $\beta_{1}$ integrin antibody AIIB2, which was developed by C.H. Damsky (19) and obtained from the Developmental Studies Hybridoma Bank at the University of Iowa, was added at $50 \mu \mathrm{g} / \mathrm{mL}$. TFPI- 1 in solution phase was typically added to the well immediately before the addition of cells. After 60 minutes of attachment and 2 washes, adherent cells were quantified by colorimetric assay (14). To determine focal adhesion kinase phosphorylation, the supernatant was gently removed from attached cells, cells were lysed in situ with reducing SDS-PAGE sample buffer, and the lysates were subjected to antiphosphotyrosine Western blotting.
Cell migration assay. Cell migration was analyzed in modified Boyden chambers (14). The lower side of the polycarbonate filter was coated with $10 \mu \mathrm{g} / \mathrm{mL}$ fibronectin, $50 \mu \mathrm{g} / \mathrm{mL}$ anti-TF mAb TF9-6B4, or 100 $\mu \mathrm{g} / \mathrm{mL}$ TFPI-1. Coated filters were placed into serumfree migration buffer containing $0.5 \% \mathrm{BSA}$, and cells were added to the upper migration chamber. VIIa (10-50 nM), Xa (50 nM), and/or heparin (5 U/mL) were added as indicated to the upper and lower chambers. Overnight migration to the lower side of the filter disk was quantified (14).

The effects of a mixed matrix on the migration was studied in a modified assay format. Filters were coated with fibronectin for more than 5 hours. To immobilize anti-TF antibody TF9-6B4, filters were transferred to a $50 \mu \mathrm{g} / \mathrm{mL}$ antibody solution for coating overnight. For simultaneous coating of fibronectin and TFPI- 1 , fibronectin-coated filters were transferred to a chamber with migration buffer that contained $1 \mu \mathrm{g} / \mathrm{mL}$ TFPI-1, which was sufficient to create a mixed matrix coat on the filter. Catalytic triad mutated, inactive VIIa Ala 344 was added at $10 \mathrm{nM}$ to both chambers to form an inactive TF-VIIa without chemotactic activity (20). J82 cells were added to the upper chamber for a migration period of 5 hours.

Immunohistochemistry. Archival tumor tissue specimens of 10 cases of invasive bladder carcinoma (grades G2 and G3) were evaluated by immunohistochemistry in areas of invasive tumor growth into the submucosa, in areas of noninvasive papillary tumor formation, and in areas of normal transitional epithelium. Sections from formalin-fixed and paraffin-embedded tissues were deparaffinized in xylene, rehydrated in graded alcohol, and blocked with $1 \%$ BSA in Tris-buffered saline (TBS). Sections were stained with specific or species- and isotype-matched primary control antibody $(10-50 \mu \mathrm{g} / \mathrm{mL})$ in TBS $(1 \%$ BSA), washed, reacted with secondary biotinylated antibody (Biogenex, San Ramon, California, USA) 1:1,000 in TBS (1\% BSA), followed by incubation with alkaline phosphatase-conjugated streptavidin. Naphthol phosphate with fast red (Sigma Chemical Co., St. Louis, Missouri, USA) was used to visualize the conjugate. Sections were counterstained with Mayer's hematoxylin. The following primary antibodies were used: anti-TF, polyclonal rabbit antibody raised against recombinant soluble TF; antiVII, polyclonal rabbit antibody raised against recombinant VII/VIIa; anti-factor X, mouse mAb's F21-4.2C (21); anti-TFPI-2, raised in rabbit by repeated intramuscular injections of the TFPI- 2 cDNA in a mammalian expression plasmid; anti-TFPI-1, rabbit antibody raised against plasma-derived TFPI-1 (kindly provided by L.V.M. Rao, University of Texas Health Center, Tyler, Texas, USA), anti-human fibrin mAb 350 (American Diagnostica, Greenwich, Connecticut, USA). Northern blot analysis. Total mRNA was extracted from surgical resection specimens for Northern blot analysis, as described previously (10). A total of $20 \mu \mathrm{g}$ of each total RNA was submitted to the analysis. The blots 
a
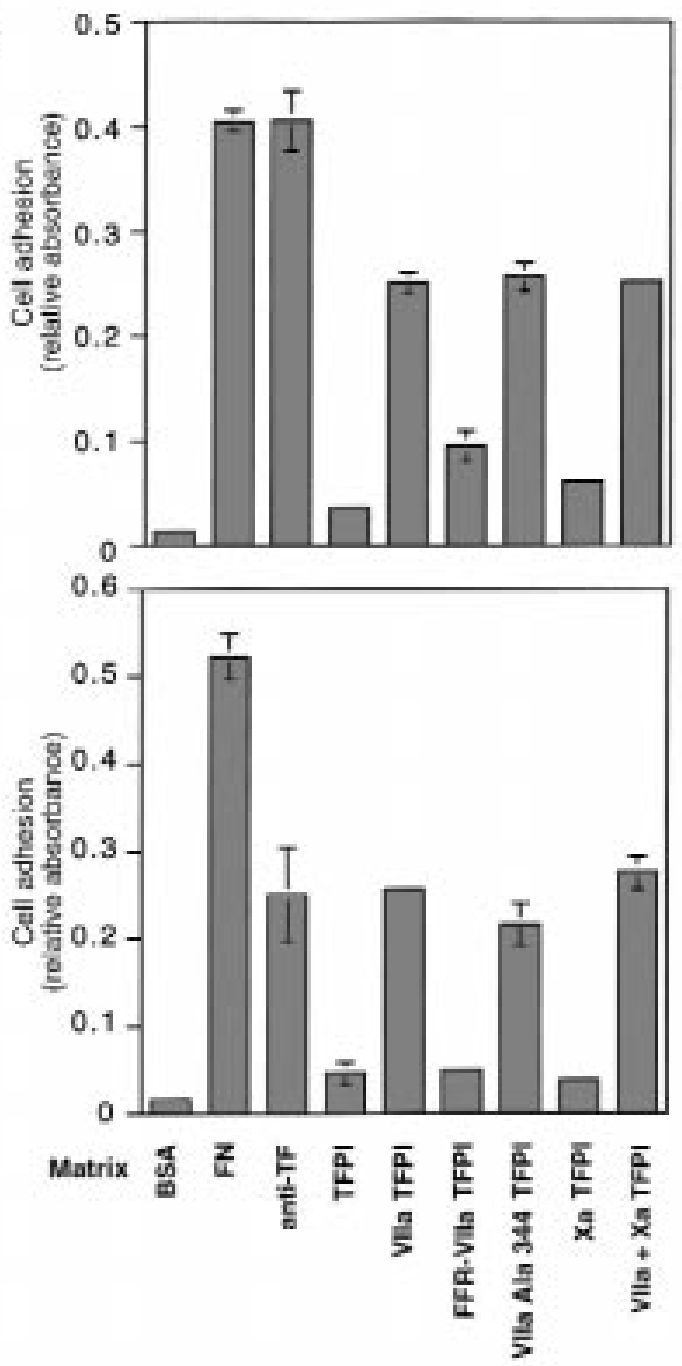

b

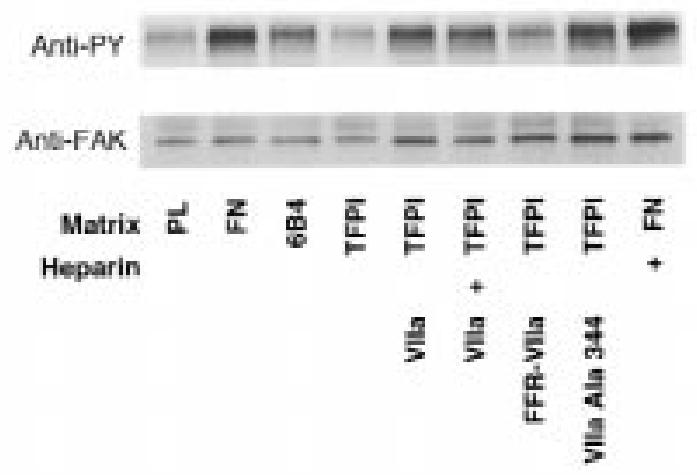

Figure 1

(a) J82 bladder carcinoma cell adhesion to (top) and migration toward (bottom) the indicated matrices (control [BSA],10 $\mu \mathrm{g} / \mathrm{mL}$ fibronectin [FN], $50 \mu \mathrm{g} / \mathrm{mL}$ antibody TF9-6B4 [anti-TF], $100 \mu \mathrm{g} / \mathrm{mL}$ recombinant TFPI-1 [TFPI]) in the presence of $10 \mathrm{nM}$ (adhesion) or $50 \mathrm{nM}$ (migration) recombinant VIla (VIla), VIla covalently active site modified with Phe-Phe-Arg chloromethylketone (FFR-VIIa), VIla rendered catalytically inactive by catalytic triad Ser 344 $\rightarrow$ Ala mutation (VIla Ala 344), or factor Xa (Xa). A total of $5 \mathrm{U} / \mathrm{mL}$ of heparin was included in all experiments to block proteoglycan-dependent attachment. (b) Phosphorylation of focal adhesion kinase (FAK) in response to adhesion to TFPI- 1 . Wells were coated with $10 \mu \mathrm{g} / \mathrm{mL}$ poly-L-lysine ( $\mathrm{PL})$ or the indicated proteins at concentrations as described for $\mathbf{a}$. Cells were allowed to adhere for 30 minutes in the presence of $5 \mathrm{U} / \mathrm{mL}$ heparin where indicated $(+)$, and with the indicated soluble proteins at $10 \mathrm{nM}$. The top panel shows an anti-phosphotyrosine (anti-PY), and the bottom panel shows an anti-FAK blot of whole-cell lysates.

ment of TF for the adhesive events, untransfected and TF-transfected CHO-K1 cells were compared in similar experiments (data not shown). Only TF-transfected cells adhered to TFPI- 1 in a VIIa-dependent, but factor Xa-independent, fashion. Adhesion of J82 cells to TFPI-1 was prevented by blocking the active site of VIIa with the covalent inhibitor Phe-Phe-Arg chloromethylketone (FFR-VIIa), but a VIIa mutant that was rendered catalytically inactive by catalytic triad Ser mutation (VIIa Ala 344) fully supported adhesion to TFPI-1 (Figure 1a). TF-VIIa-dependent adhesion to TFPI-1 was inhibited by other active site inhibitors of VIIa, by anti-TF antibodies that block VIIa binding, and by polyclonal anti-TFPI antibodies (data not shown), further demonstrating specificity. Adhesion is thus independent of catalytic activity, but requires an unoccupied active site of the TF-bound VIIa, consistent with a requirement for canonical inhibitor docking of the first Kunitz domain of TFPI-1 to the active site of VIIa. By confocal microscopy, ABP-280 was found to colocalize with TF at the contact site with TFPI-1 (data not shown), as previously shown for surrogate ligands of TF (14). Adhesion of J82 cells to immobilized ligands 


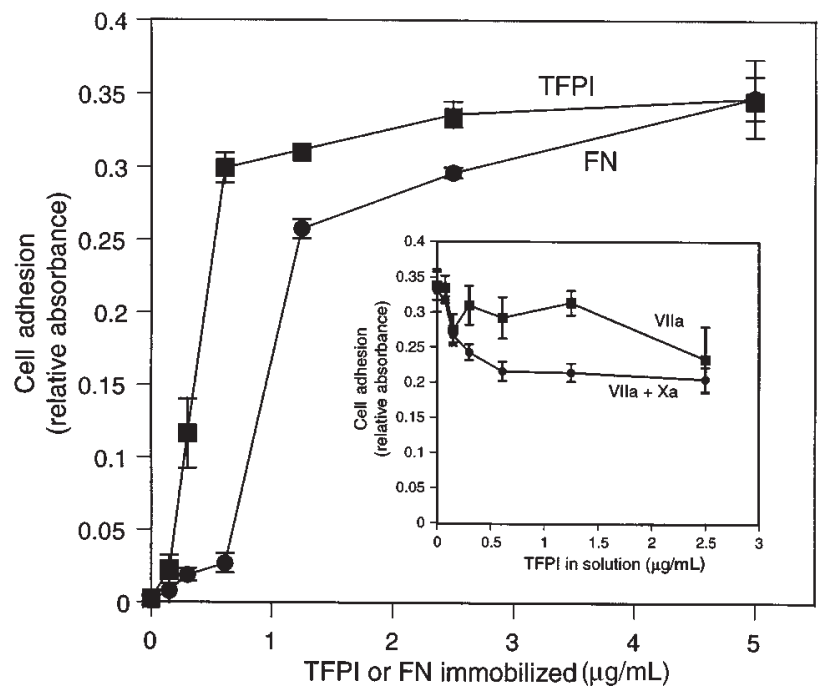

Figure 2

Potency of immobilized TFPI-1 to promote TF-VIla-mediated cell adhesion. Plates were coated with the indicated concentrations of recombinant TFPI- 1 or fibronectin. Adhesion of J82 cells was analyzed in the presence of $5 \mathrm{U} / \mathrm{mL}$ heparin; VIla $(10 \mathrm{nM})$ was added to cells for TFPI adhesion. Inset: Competition of solution-phase TFPI1. Wells were coated with $0.5 \mu \mathrm{g} / \mathrm{mL}$ TFPI-1. TFPI- 1 at the indicated concentrations was added with the cells in the presence of $10 \mathrm{nM}$ VIla or both VIla and factor Xa (Xa).

of TF, but not simple attachment to a charged surface of poly-L-lysine, induces phosphorylation of focal adhesion kinase (14). Only adhesion of J82 to TFPI- 1 in the presence of VIIa or VIIa Ala 344, but not the attachment of J82 cells presumably through cellular proteoglycans, elicited the typical phosphorylation of the 120$\mathrm{kDa}$ protein (Figure $1 \mathrm{~b}$ ). Bridging of cell-surface TF to TFPI by VIIa, but not catalytic activity of VIIa, is thus necessary for the phosphorylation of the cell migration promoting focal adhesion kinase.

TF-VIIa-dependent cell migration toward immobilized TFPI1. Haptotactic migration of J82 cells toward a gradient of immobilized TFPI- 1 was analyzed in modified Boyden chambers. Migration on TFPI- 1 in the absence of VIIa was little different from the albumin control. In the presence of VIIa, the TF-expressing J82 cells migrated on TFPI-1 similar to cells on immobilized anti-TF antibodies (Figure 1a, lower panel). In this experiment, heparin was used to compete interactions of TFPI- 1 with cell-surface proteoglycans, but experiments in the absence of heparin gave similar results. TF-VIIa-dependent migration on TFPI-1 was not influenced by factor Xa, but required an unoccupied active site of VIIa. Taken together, immobilized TFPI-1 thus supports TF-VIIa-dependent cell signaling and migration of TF-expressing human bladder carcinoma cells.

Inefficient competition by solution-phase TFPI-1 with TFVIIa-mediated cell adhesion to immobilized inbibitor. Analysis of the concentration dependence of cell adhesion to TFPI-1 demonstrated that maximal cell adhesion was observed when TFPI- 1 concentrations of $0.5-1.0 \mu \mathrm{g} / \mathrm{mL}$ were directly immobilized on the culture plastic, comparable to the concentration range of fibronectin that allows cell adhesion (Figure 2). To analyze whether TFPI-1 in solution phase competes with TFVIIa-dependent adhesion to immobilized TFPI-1, TFPI1 was included in the adhesion assay. At a close to optimal concentration of $0.5 \mu \mathrm{g} / \mathrm{mL}$ of immobilized TFPI- 1 , a 5-fold excess of solution-phase TFPI-1 produced only partial $(<40 \%)$ inhibition of cell adhesion either in the presence of VIIa or in the presence of VIIa and Xa (Figure 2 , inset). These data demonstrate that immobilization of TFPI-1, either through effects of local concentration or through conformational changes, favors complex formation with cell-associated TF-VIIa over binding to the soluble inhibitor.

Localization of the TF-VII complex at the invasive edge of bladder carcinoma. To explore whether the demonstrated mechanism of TF-VIIa-dependent migration on TFPI-1 might be of relevance for in vivo scenarios, we analyzed archival sections of 10 cases of invasive bladder carcinoma (grades G2 and G3) for the expression of components of the extrinsic coagulation pathway by immunohistochemistry. The presented data are representative for each of the 10 cases. TF was not expressed in normal mucosa, but in the submucosa, as reported previously (22). The noninvasive parts of the tumors stained weakly positive for TF, but TF expression at the invasive edge was clearly upregulated (Figure 3). Intense staining for VIIa was found only at the invasive edge of the tumor, consistent with formation of the TF-VIIa complex in an area adjacent to tumor vessels. Extravasation of plasma components was also indicated by positive staining of the invasive edge for factor $\mathrm{X}$ and for fibrin (data not shown). The latter is frequently found in tumor stroma due to angiogenesis-associated vascular hyperpermeability $(6,7)$. Examination of 13 surgical specimens of bladder carcinoma (grades G2 $[n=5]$; G3 [ $[=8]$ ) by Northern blot analysis revealed 9 of 13 samples with strong, and 3 with faint, signals for TF, but none of the samples had detectable VIIa mRNA, using a specific probe that recognized VIIa transcript in liver RNA. This supports the argument that localization of VIIa to the edge of the tumor is a consequence of extravasation from plasma, rather than de novo synthesis by the tumor cells.

The same specimens were further stained for TFPI-1 and for the closely homologous Kunitz inhibitor TFPI2 . The 2 inhibitors showed different localizations in the progression to invasive tumors (Figure 4). TFPI-2 was not detected on sections of normal bladder epithelium and papilloma. Tumor cells at the invasive edge of bladder carcinoma stained positive for TFPI-2, but the tumor-associated vessels were consistently negative for TFPI-2. In contrast, TFPI- 1 was present in an apparently extracellular localization throughout the submucosal connective tissue in normal bladder epithelium and benign papilloma (Figure 4). In invasive cancer, this pattern was lost, presumably a consequence of destruction of the normal matrix organization. The tumor neovas- 
culature that was interdispersed with the invasive edge of the tumor now stained prominently for TFPI-1. Tumor cells in proximity to the vessels also stained positive for TFPI-1, but the staining was consistently weaker than the vascular staining on the same sections.

Northern blot analysis of 13 bladder carcinoma specimens showed strong signals for TFPI-1 in 2, and for TFPI-2 in 3, cases; weak signals were found in 3 and 4 cases, respectively. Previous in vitro studies with tumor cell lines (10) suggest that the source of TFPI-2 is likely the tumor cell itself. We cannot exclude that TFPI-1 is also synthesized by the tumor cells, but the immunohistochemical data suggest that the most significant source of TFPI1 is the tumor-associated vasculature. It appears that upon local synthesis or extravasation, TFPI- 1 is retained in association with the endothelial cells, possibly creating a local concentration gradient around the tumor-infiltrating vessels. The "angiogenic switch" thus simultaneously results in TFPI- 1 expression on the tumor endothelium, as well as in extravasation of VIIa, positioning TFPI-1 on the neovasculature in close proximity to the TF-VIIa-expressing tumor cell at the invasive edge of the tumor.

Indirect immobilization of TFPI-1 through proteoglycans supports TF-VIIa-dependent adhesion. Extracellular matrix-associated proteoglycans may serve as physiological binding sites for immobilization of TFPI-1. TF-VIIa-mediated cell adhesion was studied on plates coated with heparin that can bind the basic $\mathrm{COOH}$ terminus and the third Kunitz domain of TFPI-1. Blocking with BSA prevented direct binding of TFPI- 1 to the plastic

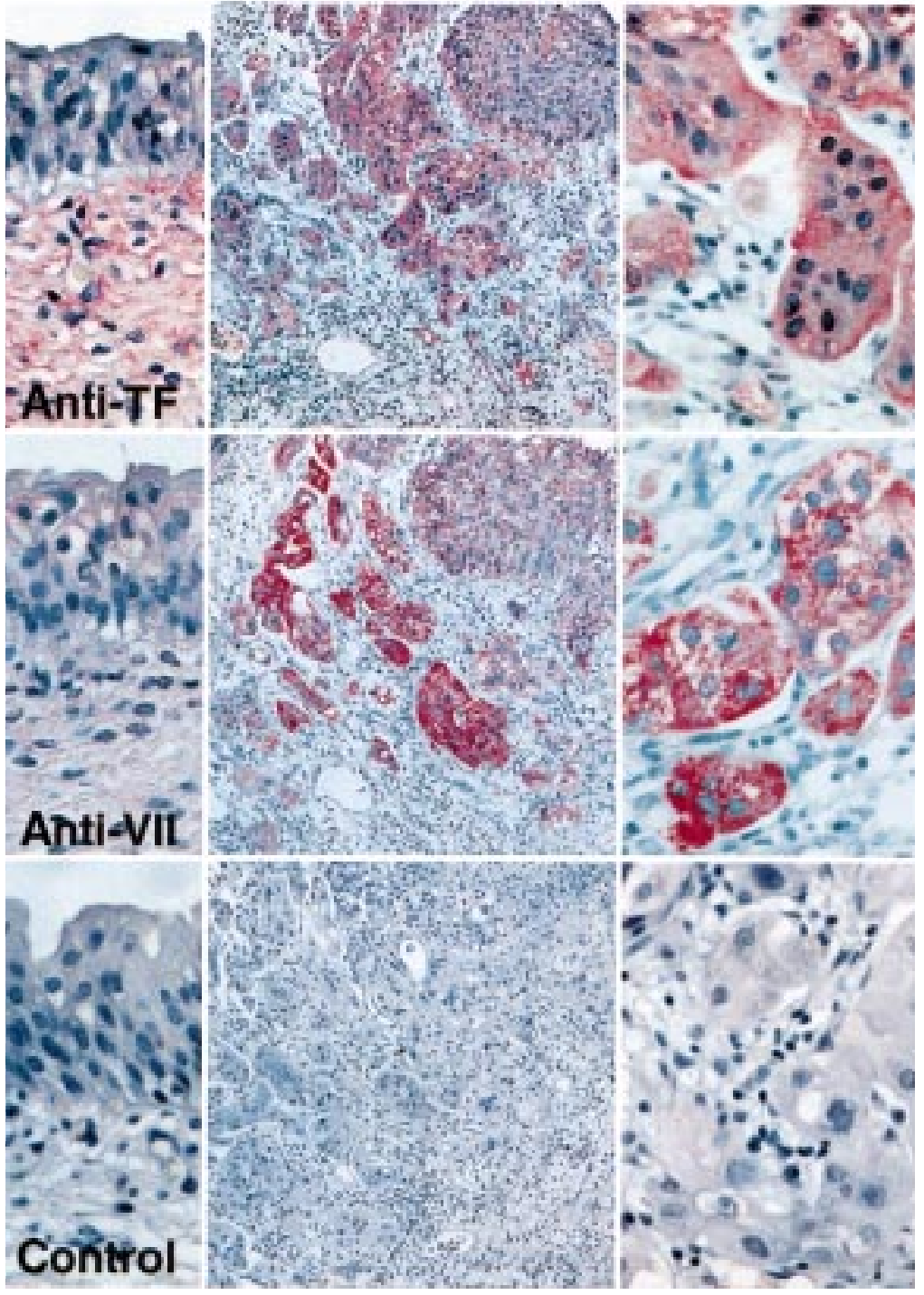

Figure 3

Immunohistochemical detection of TF and VIla in invasive bladder carcinoma. The left panels show areas of normal transitional epithelium $(\times 400)$ adjacent to the invasive areas of the tumor shown as an overview in the middle $(\times 100)$ and at high magnification in the right panels $(\times 400)$. The control is staining with anti-TF antibody in the presence of $100 \mu \mathrm{g} / \mathrm{mL}$ soluble TF to demonstrate specificity. surface. TFPI- 1 added together with the cell suspension potently promoted VIIa-dependent cell adhesion to heparin-coated plates (Figure 5), and maximal adhesion was observed at approximately 100 $\mathrm{ng} / \mathrm{mL}$, corresponding to physiological plasma concentrations of TFPI-1 (23). When heparin-coated wells were preincubated with TFPI-1 for 1 hour and the solution was removed before the addition of cells in the presence of VIIa, equal or even more potent adhesion was observed (Figure 5), suggesting that TFPI-1 rapidly bound to the immobilized heparin to allow subsequent cell adhesion. These data demonstrate that surface association of TFPI-1 through immobilized glycans promotes cell adhesion mediated by TFVIIa. Notably, attachment of cells was not observed in the absence of VIIa, indicating that the proteoglycan binding sites on TFPI-1 were completely masked upon binding to the immobilized heparin. VIIa binding to cell surface TF was sufficient to promote adhesion to immobilized TFPI-1, based on experiments in which the suspended cells were preincubated with VIIa followed by removal of excess solution phase protease before plating (data not shown). Inclusion of 10 $\mu \mathrm{g} / \mathrm{mL}$ TFPI- 1 together with $10 \mathrm{nM}$ VIIa in a similar preincubation experiment did not promote adhesion to heparin, arguing against an adhesive pathway in which formation of the TF-VIIa/TFPI-1 complex on the cell surface triggers the subsequent adhesion to proteoglycans in the extracellular matrix. Rather, heparin-proteoglycan-dependent immobilization of TFPI-1 appears to promote an inhibitor conformation that enhances adhesive interaction with the cell surface-expressed TF-VIIa complex. 
Cooperation of TFPI-1 with adhesion to and migration on integrin matrix. Although invasive areas of tumors are characterized by matrix degradation and turnover, extracellular matrix proteins that mediate cell adhesion and migration through integrins likely are present. To address whether the TF-VIIa-dependent mechanism of cell adhesion to TFPI-1 may be of physiological relevance in the presence of integrin matrix, cell adhesion on mixed matrix containing equal concentrations of TFPI- 1 and fibronectin was analyzed in the presence and absence of VIIa. VIIa did not enhance cell adhesion
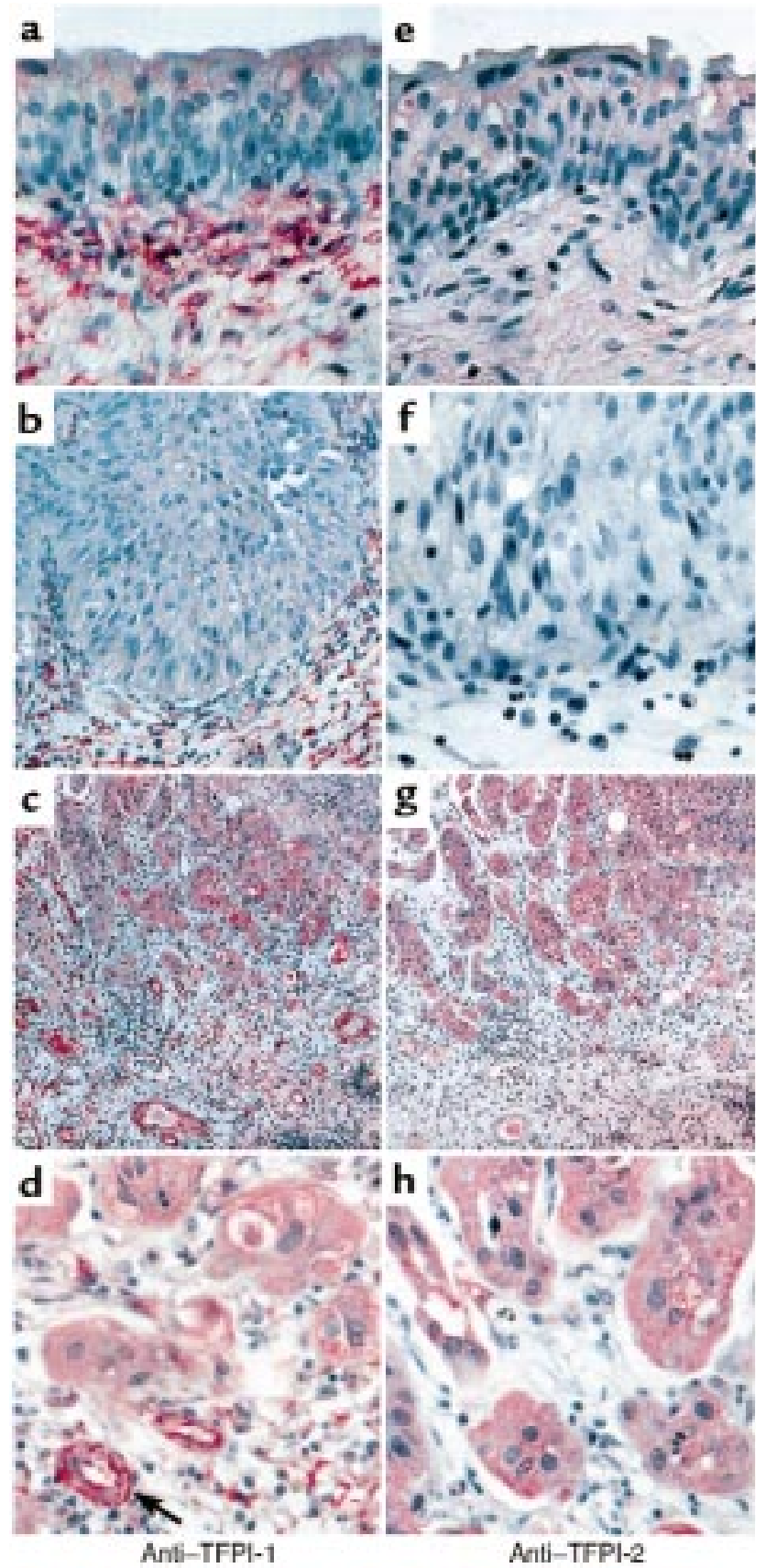

Figure 4

Staining for TFPI-1 (a-d) and TFPI-2 (e-h) of normal epithelium (a, e), noninvasive tumor $(\mathbf{b}, \mathbf{f})$, and invasive bladder carcinoma (c, d, g, h). Magnification was $\times 400$, except for overviews taken at $\times 100$. at high concentrations ( $>1 \mu \mathrm{g} / \mathrm{mL}$ ) of fibronectin. Whereas cell adhesion to fibronectin rapidly decreased upon lowering of this extracellular matrix protein (Figure $6 \mathrm{a}$ ), similar concentrations of TFPI-1 still supported VIIa-dependent cell adhesion (Figure 6a), arguing that TF-VIIa-mediated cell adhesion is effective when the concentrations of typical integrin ligands are reduced. In the presence of an inhibitory anti- $\beta_{1}$ integrin antibody that reduced adhesion to background levels in the absence of VIIa, adhesion to TFPI-1 through TF-VIIa efficiently compensated for the loss of integrin function (Figure 6b). However, VIIa did not completely reverse the loss of integrin-dependent adhesion, indicating a functional cooperation of TF-VIIa and integrinmediated adhesion to this composite matrix.

A cooperation of TF with integrins in cell migration was directly tested in haptotactic migration assays. The lower side of the migration chambers was coated first with fibronectin at low concentrations that poorly supported migration. Additional coating with anti-TF antibody greatly enhanced migration, whereas only inefficient migration was observed with antibody alone in this short-term (5-hour) assay format (Figure 7a). Migration on the composite matrix consisting of fibronectin and anti-TF antibody was inhibited by soluble TF as well as inhibitory anti- $\beta 1$ integrin antibody, demonstrating cooperation between the 2 adhesive pathways. Addition of TFPI-1 to filters precoated with low concentrations of fibronectin also enhanced migration in the presence of catalytically inactive VIIa, but only inefficient migration was observed when VIIa or TFPI-1 was added separately (Figure 7b). Cooperation of integrins with TF-VIIa-mediated migration is further demonstrated by the dose-dependent enhancement by increasing fibronectin concentrations (Figure 7c). Thus, TF-VIIa-mediated binding to matrix-associated TFPI- 1 plays a critical role in cell migration when the concentration of integrin-binding extracellular matrix components is reduced.

\section{Discussion}

This study demonstrates that the Kunitz-type inhibitor TFPI-1 supports TF-dependent cell adhesion, migration, and intracellular signaling. TF's physiological protease ligand VIIa is necessary for migration toward TFPI-1, but catalytic activity of the protease is not required. This enhanced migration thus differs from the previously demonstrated stimulation of cell motility by VIIa that required catalytic activity $(20,24)$. A proteaseactivated receptor for VIIa has been suggested from studies of VIIa-dependent MAP kinase activation (25), and VIIa-dependent protease signaling may trigger a proteolytic pathway similar to the well-characterized thrombin receptor pathway that also influences cytoskeleton dynamics. In the adhesion to TFPI-1, VIIa serves a distinct role, which is bridging of the cell-surface receptor TF to the immobilized inhibitor. Immobilized heparin, a surrogate for extracellular matrix-associated heparan-sulfated proteoglycans, was found to 


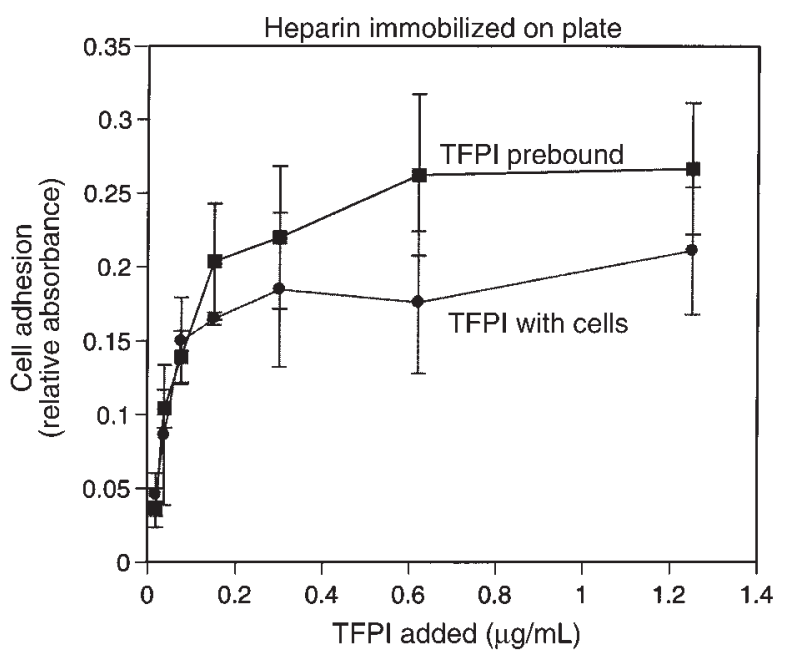

Figure 5

Heparin-mediated immobilization of TFPI-1 promotes cell adhesion. Wells were coated with $5 \mathrm{U} / \mathrm{mL}$ heparin followed by blocking with BSA. The indicated concentrations of TFPI- 1 were either added together with the cell suspension or preincubated with the heparincoated well for 1 hour, followed by removal of unbound inhibitor before the addition of the cell suspension. The cell adhesion buffer contained $10 \mathrm{nM} \mathrm{VIla}$, and adhesion was determined after 1 hour.

allow binding of TFPI- 1 in a conformation that favored the interaction with the TF-VIIa complex expressed on the tumor cell surface. In addition, incorporation of TFPI- 1 into a matrix that contained low concentrations of ligands for integrin adhesion receptors enhanced the adhesion and migration of TF-VIIa-expressing tumor cells. These data provide evidence for a functional cooperation between TF and integrins in cell adhesion and migration, in particular when the concentration of integrin-specific extracellular matrix protein is reduced in scenarios of matrix remodeling.

The extracellular matrix undergoes massive degradation in tumor invasion. In human bladder cancer progression, intense staining for TFPI- 1 was found on the luminal and abluminal side of the vascular endothelium. This may create a TFPI- 1 gradient in the perivascular tumor stroma, supporting TF-VIIa-dependent tumor cell migration toward the angiogenic tumor vasculature. It is notable that solution-phase TFPI- 1 was a poor competitor for TF-VIIa-mediated adhesion to immobilized TFPI-1 in the in vitro experiments, indicating that matrix-enriched TFPI-1 may serve adhesive functions even in the presence of fluid-phase TFPI- 1 that extravasated from the tumor vasculature. The embryonic lethality associated with genetic deletion of the first Kunitz-domain of TFPI- 1 in mice (26) poses an experimental challenge to test rigorously the role of TFPI- 1 in experimental models of tumor progression. Specific inhibitors, such as species-specific high-affinity $\mathrm{mAb}$ 's to the first Kunitz domain of TFPI-1, may eventually allow testing whether vessel associated TFPI- 1 is necessary for tumor progression and invasion, as suggested by the presented data that illustrate expression of TFPI- 1 in this specific context of matrix remodeling.

Moreover, the TF-VIIa complex was found to localize to the invasive edge of human bladder carcinoma in vivo. Because bladder carcinomas did not express detectable levels of VIIa mRNA, we infer that the tumor-associated VIIa extravasates from the plasma in the context of vascular hyperpermeability induced by VEGF, known to be upregulated in bladder carcinoma (27). Extravasated plasma components frequently
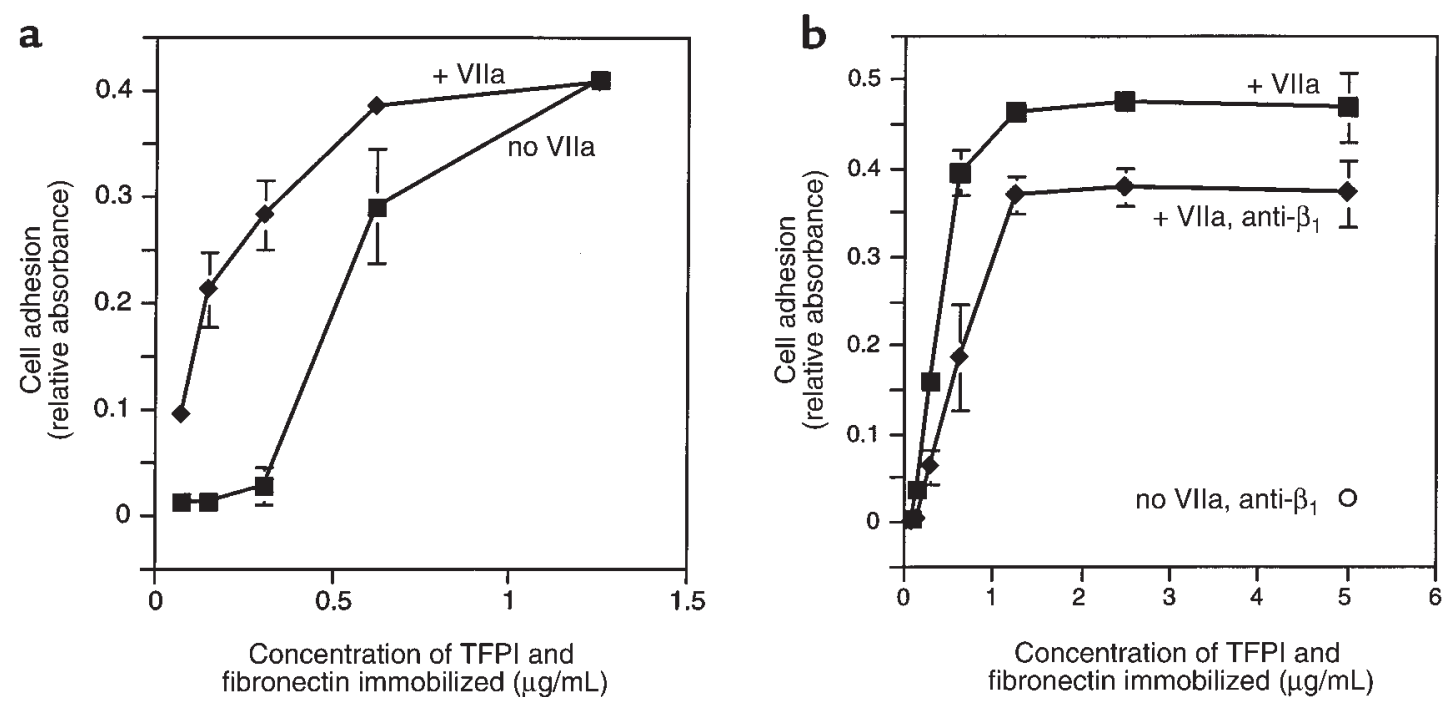

Figure 6

Adhesion on composite matrix. Wells were coated with equal concentrations of fibronectin and TFPI-1, as indicated on the $x$ axis. (a) Effect of $10 \mathrm{nM}$ VIla on the adhesion to the composite matrix. The adhesion buffer contained $5 \mathrm{U} / \mathrm{mL}$ heparin to block proteoglycan-dependent cell adhesion to TFPI-1. (b) Effect of function-blocking anti- $\beta_{1}$ integrin antibody on the binding to composite matrix. The antibody at $50 \mu \mathrm{g} / \mathrm{mL}$ completely blocked adhesion in the absence of VIla (anti- $\beta_{1}$, no VIla). Adhesion to the composite matrix in the presence $\left(+\right.$ anti- $\left.\beta_{1}\right)$ or absence of the blocking antibody was analyzed at various concentrations of immobilized matrix and in the presence of $10 \mathrm{nM} \mathrm{VIla} \mathrm{and} 5 \mathrm{U} / \mathrm{mL}$ heparin. 
serve matrix degradation through receptor-orchestrated protease cascades, thus functionally contributing to the well-established link of the angiogenic switch of tumors with tumor cell invasion and metastasis $(28,29)$. The presented data for TF suggest a novel mechanism by which a protease receptor may participate in tumor progression. In the case of TF, the critical function of the protease is to bridge the adhesion/migration-supporting cell surface receptor to extracellular inhibitors for directed invasion or possibly for the creation of cell-cell contacts under conditions of vascular hyperpermeability.

The essential function of TFPI- 1 in regulating the proteolytic function of TF-VIIa is well established (26). Although TFPI-1 has clearly been shown to bind and inhibit TF-VIIa in the absence of factor Xa (30), factor $\mathrm{Xa}$ is critical for the potency of inhibition of TF-VIIa by TFPI-1. In contrast, TF-mediated adhesion on and migration toward TFPI-1 solely depended on the presence of VIIa. This finding indicates a fundamental difference in how the TF-VIIa complex binds to TFPI-1 associated with extracellular matrices. The most reasonable explanation for the enhanced complex formation with TF-VIIa upon matrix binding of TFPI- 1 is a conformational change that allows favorable presentation of the first Kunitz-type domain for binding to the catalytic site of VIIa. Interactions between the charged termini of TFPI-1 may influence the fluid-phase conformation of the inhibitor (31) and binding of the basic $\mathrm{COOH}$-terminus to matrix proteoglycans could trigger a significant conformational change that exposes the first Kunitz domain of TFPI-1.

Heparin binding to TFPI-2 also enhanced the interaction of TFPI-2's first Kunitz domain with TF-VIIa (11). TFPI-2 is known to preferentially sequester into the extracellular matrix of cultured cells (32), possibly indicating that this inhibitor also functions in the regulation of cell adhesion. This suggests a more general paradigm for Kunitz-type inhibitors that may serve dual roles in regulating proteolysis in fluid phase, as well as serving as adhesive ligands upon matrix association. Although the presented example of bladder cancer invasion suggests a functional role for TFPI-1, different Kunitz-type inhibitors may function in other scenarios in which TF appears to support cell adhesion and migration, such as metastatic tumor cell homing and extravasation (13) or reverse transmigration of monocyte/macrophages through the endothelium (33). Endothelial cells synthesize amyloid $\beta$ protein precursor that interacts with VIIa through its Kunitz domain (34); and deposition of TFPI-2 into the endothelial matrix is upregulated by inflammatory cytokines (35). On tumor or inflammatory cells that extravasate from the blood, the TF-VIIa complex is formed due to expo- a

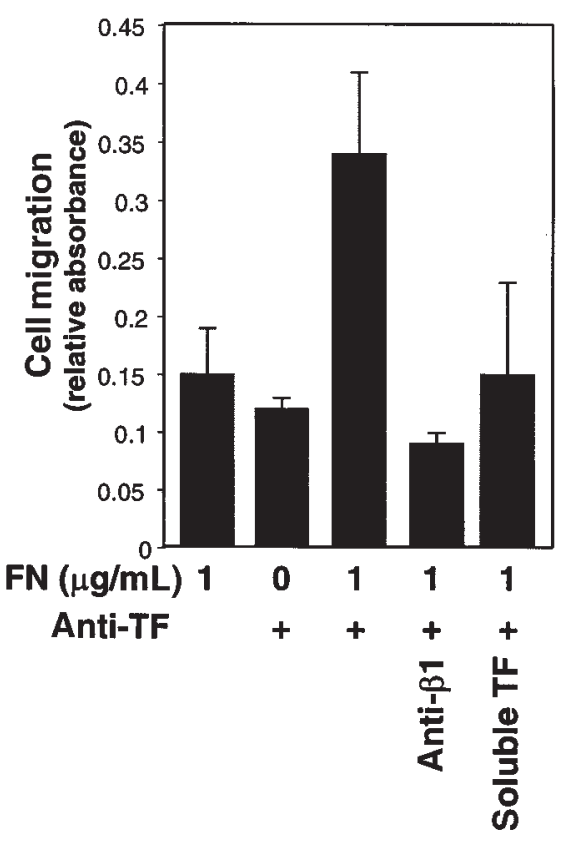

b

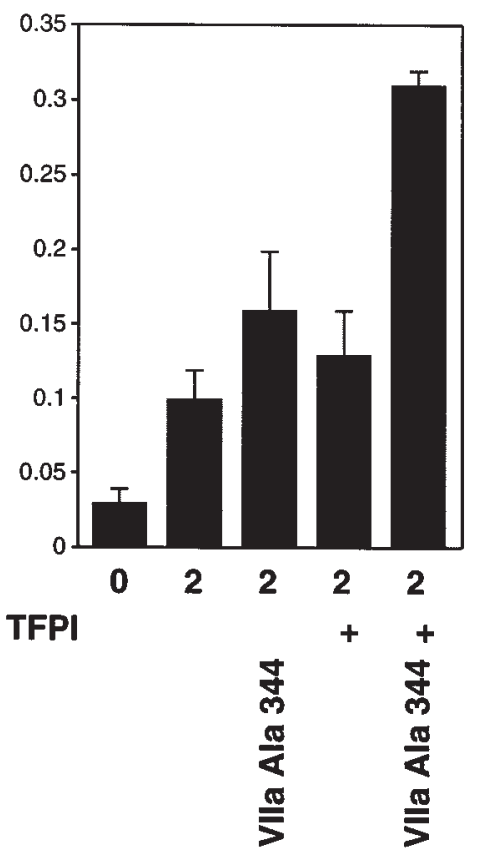

C

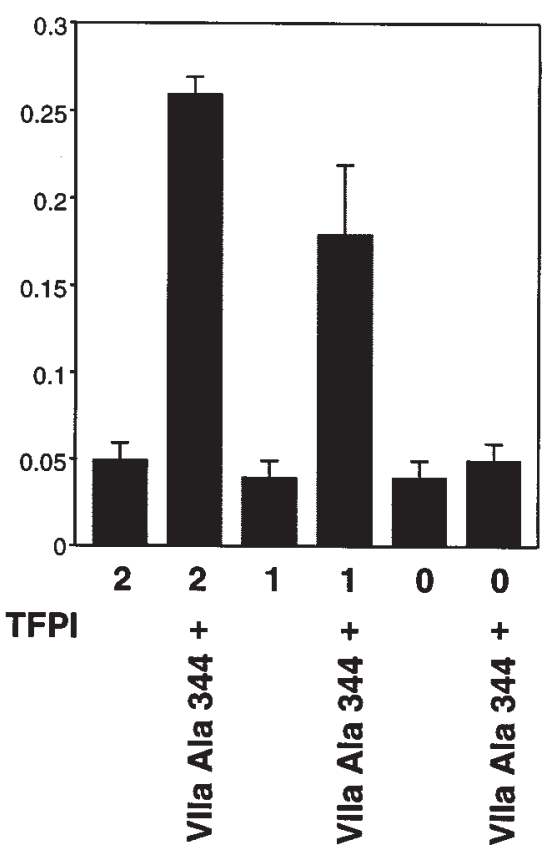

Figure 7

Cooperation of TF with integrins in the migration of $\mathrm{J} 82$ bladder carcinoma cells. The lower side of the migration filter was coated with the indicated concentration of fibronectin (FN) followed by a second coating with anti-TF antibody TF9-6B4 $(50 \mu \mathrm{g} / \mathrm{mL})$ or TFPI $(1 \mu \mathrm{g} / \mathrm{mL})$, as indicated. J82 cell migration was quantified after 5 hours. Catalytically inactive VIla Ala 344 was present at $10 \mathrm{nM}$ in the upper and lower migration chamber, where indicated. (a) Enhanced migration on fibronectin/anti-TF-coated filters is blocked with soluble TF (100 $\mu \mathrm{g} / \mathrm{mL}$ ) or the inhibitory anti- $\beta_{1}$ integrin antibody AIIB2 $(50 \mu \mathrm{g} / \mathrm{mL})$, both present in the upper and lower compartments of the migration chamber. (b) VIla is required for enhanced migration on TFPI/fibronectin-coated surfaces. (c) TFPI/VIla enhanced migration depends on the amount of immobilized fibronectin. 
sure to plasma components, and these cells are capable of using the Kunitz-type inhibitors associated with the subendothelial matrix. The protease-mediated bridging to extracellular inhibitors may thus represent a general mechanism by which the extracellular domain of TF is engaged to promote the intracellular signaling events that support cell migration.

\section{Acknowledgments}

We thank Jennifer Royce, Dorothy Markowitz, and Pablito Tejada for technical assistance; A. Creasy for recombinant TFPI-1; and L.V.M. Rao for anti-TFPI-1 antibody. This work was supported by grants from the National Institutes of Health (PO1-HL 16411 and RO1-HL60742). W. Ruf is an Established Investigator of the American Heart Association.

1. Mignatti, P., and Rifkin, D.B. 1993. Biology and biochemistry of proteinases in tumor invasion. Physiol. Rev. 73:161-195.

2. Johnsen, M., Lund, L.R., Romer, J., Almholt, K., and Dano, K. 1998. Cancer invasion and tissue remodeling: common themes in proteolytic matrix degradation. Curr. Opin. Cell Biol. 10:667-671.

3. Powell, W.C., and Matrisian, L.M. 1996. Complex roles of matrix metalloproteinases in tumor progression. Curr. Top. Microbiol. Immunol. 213:1-22

4. Ruf, W., and Edgington, T.S. 1994. Structural biology of tissue factor, the initiator of thrombogenesis in vivo. FASEB J. 8:385-390.

5. Callander, N.S., Varki, N., and Rao, L.V.M. 1992. Immunohistochemical identification of tissue factor in solid tumors. Cancer. 70:1194-1201.

6. Shoji, M., et al. 1998. Activation of coagulation and angiogenesis in cancer. Immunohistochemical localization in situ of clotting proteins and vascular endothelial growth factor in human cancer. Am. J. Pathol. 152:399-411.

7. Dvorak, H.F., Brown, L.F., Detmar, M., and Dvorak, A.M. 1995. Vascular permeability factor/vascular endothelial growth factor, microvascular hyperpermeability, and angiogenesis. Am. J. Pathol. 146:1029-1038.

8. Broze, G.J. 1995. Tissue factor pathway inhibitor and the revised theory of coagulation. Annu. Rev. Med. 46:103-112.

9. Sprecher, C.A., Kisiel, W., Mathewes, S., and Foster, D.C. 1994. Molecular cloning, expression, and partial characterization of a second human tissue-factor-pathway inhibitor. Proc. Natl. Acad. Sci. USA. 91:3353-3357.

10. Miyagi, Y., et al. 1994. cDNA cloning and mRNA expression of a serine proteinase inhibitor secreted by cancer cells: identification as placental protein 5 and tissue factor pathway inhibitor-2. J. Biochem. (Tokyo). 116:939-942.

11. Petersen, L.C., et al. 1996. Inhibitory properties of a novel human Kunitz-type protease inhibitor homologous to tissue factor pathway inhibitor. Biochemistry. 35:266-272.

12. Bromberg, M.E., Konigsberg, W.H., Madison, J.F., Pawashe, A., and Garen, A. 1995. Tissue factor promotes melanoma metastasis by a path way independent of blood coagulation. Proc. Natl. Acad. Sci. USA. 92:8205-8209.

13. Mueller, B.M., and Ruf, W. 1998. Requirement for binding of catalytically active factor VIIa in tissue factor dependent experimental metastasis. J. Clin. Invest. 101:1372-1378.

14. Ott, I., Fischer, E.G., Miyagi, Y., Mueller, B.M., and Ruf, W. 1998. A role for tissue factor in cell adhesion and migration mediated by interaction with actin binding protein 280. J. Cell Biol. 140:1241-1253.
15. Cunningham, C.C., et al. 1992. Actin-binding protein requirement for cortical stability and efficient locomotion. Science. 255:325-327.

16. Wei, Y., et al. 1994. Identification of the urokinase receptor as an adhesion receptor for vitronectin. J. Biol. Chem. 269:32380-32388.

17. Brooks, P.C., et al. 1996. Localization of matrix metalloproteinase MMP2 to the surface of invasive cells by interaction with integrin $\alpha v \beta 3$. Cell. 85:683-693.

18. Diaz-Collier, J.A., et al. 1994. Refold and characterization of recombinant tissue factor pathway inhibitor expressed in Escherichia coli. Thromb. Haemost. 71:339-346.

19. Werb, Z., Tremble, P.M., Behrendtsen, O., Crowley, E., and Damsky, C.H. 1989. Signal transduction through the fibronectin receptor induces collagenase and stromelysin gene expression. J. Cell Biol. 109:877-890.

20. Sato,Y., et al. 1997. Tissue factor pathway inhibitor inhibits aortic smooth muscle cell migration induced by tissue factor/factor VIIa complex. Thromb. Haemost. 78:1138-1141.

21. Sevinsky, J.R., Rao, L.V.M., and Ruf, W. 1996. Ligand-induced protease receptor translocation into caveolae: a mechanism for regulating cell surface proteolysis of the tissue factor-dependent coagulation pathway. $J$. Cell Biol. 133:293-304.

22. Drake, T.A., Morrissey, J.H., and Edgington, T.S. 1989. Selective cellular expression of tissue factor in human tissues. Am. J. Pathol. 134:1087-1097.

23. Novotny, W.F., Brown, S.G., Miletich, J.P., Rader, D.J., and Broze, G.J., Jr. 1991. Plasma antigen levels of the lipoprotein-associated coagulation inhibitor in patient samples. Blood. 78:387-393.

24. Müller, M., et al. 1999. Localization of tissue factor in actin-filament-rich membrane areas of epithelial cells. Exp. Cell Res. 248:136-147.

25. Sorensen, B.B., et al. 1999. Factor VIIa-induced p44/42 mitogen-activated protein kinase activation requires the proteolytic activity of factor VIIa and is independent of the tissue factor cytoplasmic domain. J. Biol. Chem. 274:21349-21354.

26. Huang, Z.-F., Higuchi, D., Lasky, N., and Broze, G.J., Jr. 1997. Tissue factor pathway inhibitor gene disruption produces intrauterine lethality in mice. Blood. 90:944-951.

27. Brown, L.F., et al. 1993. Increased expression of vascular permeability factor (vascular endothelial growth factor) and its receptors in kidney and bladder carcinomas. Am. J. Pathol. 143:1255-1262.

28. Skobe, M., Rockwell, P., Goldstein, N., Vosseler, S., and Fusenig, N.E. 1997. Halting angiogenesis suppresses carcinoma cell invasion. Nat. Med. 3:1222-1227.

29. Zetter, B.R. 1998. Angiogenesis and tumor metastasis. Annu. Rev. Med. 49:407-424.

30. Callander, N.S., et al. 1992. Mechanisms of binding of recombinant extrinsic pathway inhibitor (rEPI) to cultured cell surfaces. Evidence that rEPI can bind to and inhibit factor VIIa-tissue factor complexes in the absence of factor Xa. J. Biol. Chem. 267:876-882.

31. Wesselschmidt, R., Likert, K., Huang, Z., MacPhail, L., and Broze, G.J., Jr. 1993. Structural requirements for tissue factor pathway inhibitor interactions with factor Xa and heparin. Blood Coagul. Fibrinolysis. 4:661-669.

32. Rao, C.N., et al. 1996. Extracellular matrix-associated serine protease inhibitors $\left(\mathrm{M}_{\mathrm{r}} 33,000,31,000\right.$, and 27,000$)$ are single-gene products with differential glycosylation: cDNA cloning of the 33-kDa inhibitor reveals its identity to tissue factor pathway inhibitor-2. Arch. Biochem. Biophys. 335:82-92.

33. Randolph, G.J., Luther, T., Albrecht, S., Magdolen, V., and Muller, W.A. 1998. Role of tissue factor in adhesion of mononuclear phagocytes to and trafficking through endothelium in vitro. Blood. 92:4167-4177.

34. Dennis, M.S., and Lazarus, R.A. 1994. Kunitz domain inhibitors of tissue factor-factor VIIa. I. Potent inhibitors selected from libraries by phage display. J. Biol. Chem. 269:22129-22136.

35. Iino, M., Foster, D.C., and Kisiel, W. 1998. Quantification and characterization of human endothelial cell-derived tissue factor pathway inhibitor-2. Arterioscler. Thromb. Vasc. Biol. 18:40-46. 